UCRL-JC-112347

PREPRINT

\title{
Molecular Dynamics Modeling of the Mechanical Behavior of Metallic Multilayers
}

\author{
James Belak and David B. Boercker
}

This paper was prepared for submittal to the proceedings of the

Spring American Chemical Society Meeting

Denver, $\mathrm{CO}$

March 28-April 2, 1993

April 1993

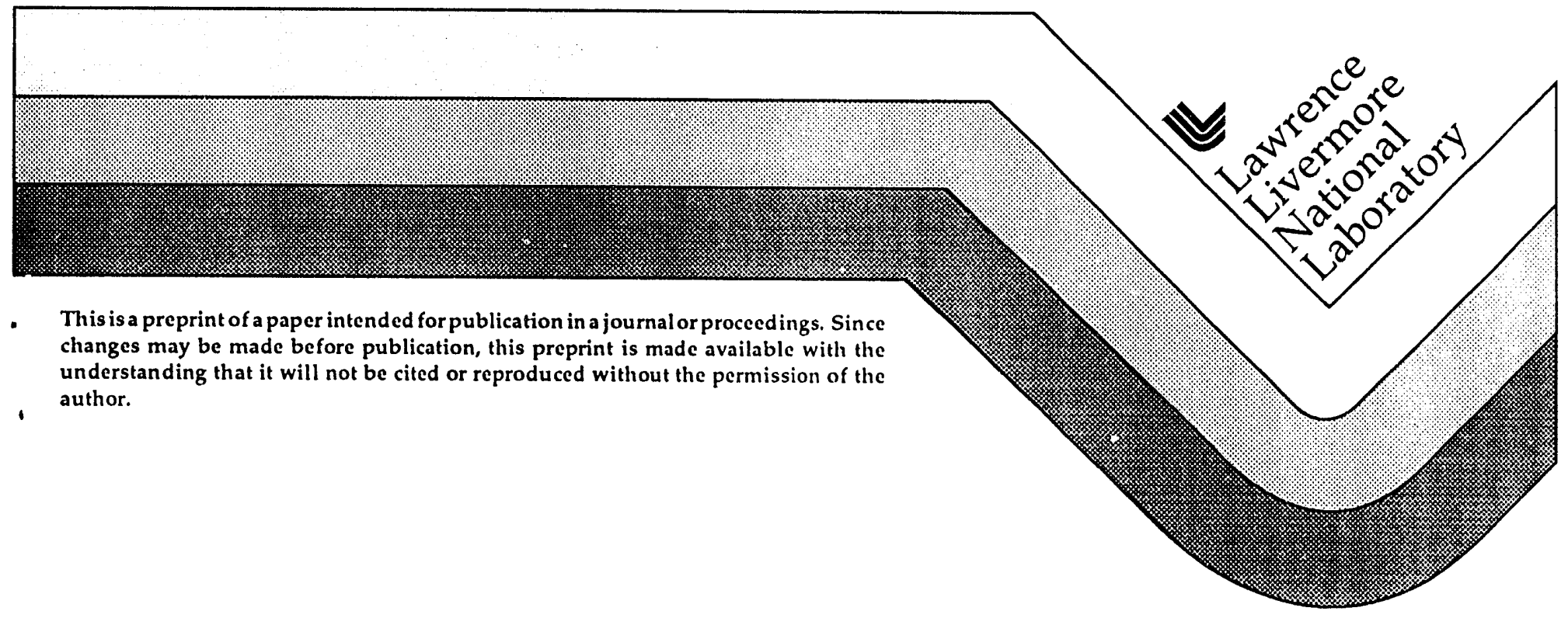




\section{DISCLAIMER}

This document was prepared as an account of work sponsored by an agency of the United States Government. Neither the United States Government nor the University of California nor any of their employees, makes any warranty, express or implied, or assumes any legal liability or responsibility for the accuracy, completeness, or usefulness of any information, apparatus, product, or process disclosed, or represents that its use would not infringe privately owned rights. Reference herein to any spedific commercial product, process, or service by trade name, trademark, manufacturer, or otherwise, does not necessarily constitute or imply its endorsement, recommendation, or favoring by the United States Government or the University of California. The views and opinions of authors expressed herein do not necessarily state or reflect those of the United States Government or the University of California, and shall not be used for advertising or product endorsement purposes. 
MOLECULAR DYNAMICS MODELING OF THE MECHANICAL BEHAVIOR OF METALLIC MULTILAYERS*

James Belak and David B. Boercker

Lawrence Livermore National Laboratory, Livermore, CA 94550

\begin{abstract}
Constant-stress molecular dynamics simulations are used to study the mechanical properties of equal concentration $\mathrm{Cu}-\mathrm{Ni}$ (111) metallic multilayers of repeat lengths $0.4-5.0 \mathrm{~nm}$. Uniaxial stress is applied along the close-packed and perpendicular to the close-packed directions within the (111) plane. The observed elastic moduli do not display a supermodulus effect as observed in experimental bulge tests for the biaxial modulus. However, both the average interlayer spacing and the out-of-plane Poisson ratio display anomalous effects for multilayer repeat lengths below about two nanometers.
\end{abstract}

\title{
INTRODUCTION
}

Metallic multilayers are nano-engineered materials in which the repeat layer spacing and intralayer composition can be controlled with sub-nanometer precision. These materials display unique mechanical, thermal, electrical and optical properties.[1] Early bulge test experiments on $\mathrm{Cu}-\mathrm{Ni}$ (111) multilayers displayed an anomalous supermodulus effect for the biaxial elastic modulus Y(111).[2] Recent nanoindentation experiments, however, do not display this effect.[3] The purpose of the present paper is to examine the mechanical properties, in particular in-plane elastic moduli, of equal concentration $\mathrm{Cu}-\mathrm{Ni}$ (111) multilayers using the molecular dynamics computer simulation method.

\section{THEORETICAL}

Ideal $\mathrm{Cu}-\mathrm{Ni}(111)$ multilayers are constructed from an fcc $(\mathrm{ABC})$ lattice as follows. The $\hat{z}$ axis of the basic unit cell is along the [111] direction in the cubic fcc lattice. The $\hat{x}$ axis is along the close-packed direction within the (111) plane with edge length equal to the nearest neighbor spacing. The $\hat{y}$ axis is also in the (111) plane, orthogonal to the $\hat{x}$ axis. Three simulation cell sizes are considered, all as nearly cubic as possible; (1) $\mathrm{N}=1440$ atoms in 12 layers, (2) $\mathrm{N}=$ 4860 atoms in 18 layers, and (3) $\mathrm{N}=11520$ atoms in 24 layers. It is important that both the multilayer repeat length and the fcc repeat length ( 3 layers) be commensurate with the number of layers in the simulation cell. For all of the simulation results presented here, the system is equilibrated for a period of 2000 time steps $(\Delta t=5 \mathrm{fs})$ at room temperature after which averages are taken for a period of 10000 steps.

The embedded-atom method[4] potential model due to Johnson[5] is employed in the present study. Johnson[6] has shown that, for the embedding density functions to be transferable and the embedding energy to be transformation invariant, the two-body potential for the alloy must take the form:

$$
\varphi_{A B}=\frac{1}{2}\left\{\frac{f_{A}}{f_{B}} \varphi_{B B}+\frac{f_{B}}{f_{A}} \varphi_{A \Lambda}\right\}
$$

where $\varphi_{A A}$ and $\varphi_{B B}$ are the two-body potentials between separate species $A$ and $B$. The functions $f_{A}$ and $f_{B}$ are the embedding densities due to species $\mathrm{A}$ and $\mathrm{B}$ respectively.

The computer experiment consists of applying a given stress and observing the response of the simulation cell during a variable shape simulation.[7] In this work, equations of motion equivalent to those derived by Cleveland [8] are used. 


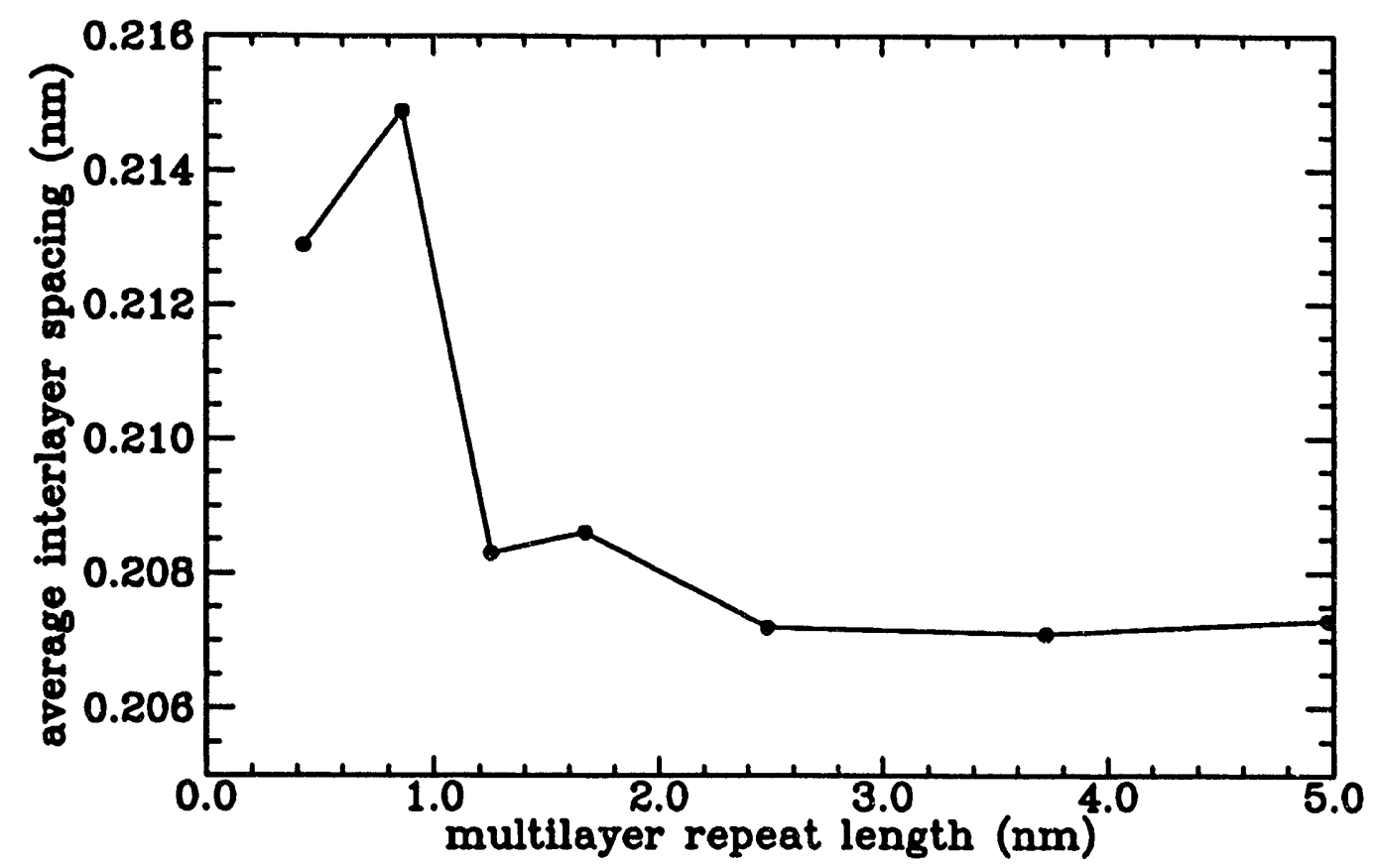

Figure 1. The calculated average interlayer spacing for equal concentration $\mathrm{Cu}-\mathrm{Ni}$ (111) multilayers as a function of multilayer repeat length. The statistical error in the calculation is comparable to the symbol size.

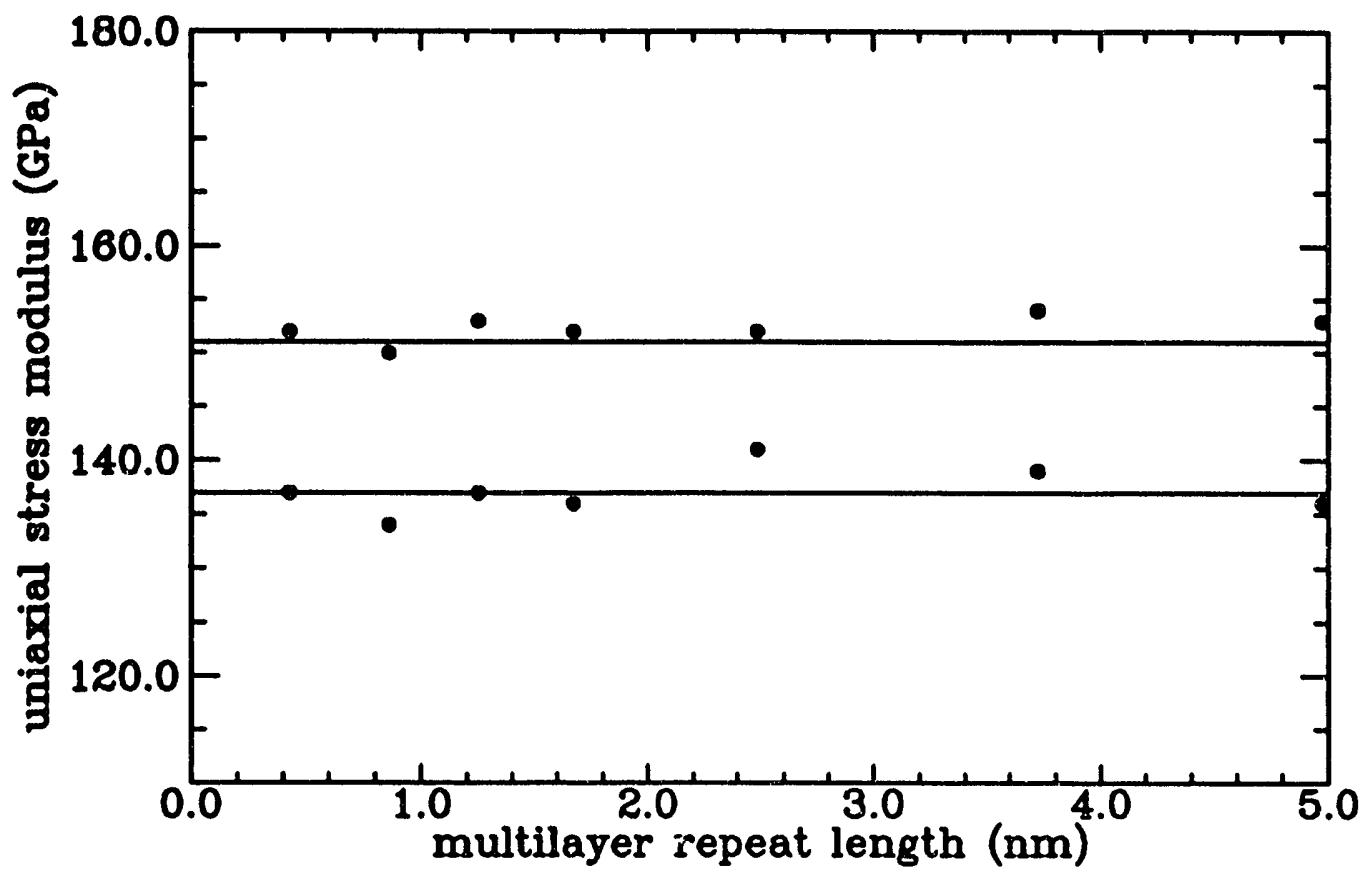

Figure 2. The calculated uniaxial stress modulus for stress along the $\mathrm{x}$ axis (lower curve) and along the y axis (upper curve) for equal concentration $\mathrm{Cu}-\mathrm{Ni}$ (111) multilayers as a function of multilayer repeat length. The straight lines are drawn as a guide to the eye. The statistical error in the calculation is somewhat larger than the symbol size (about a few percent). The fluctuations are not significant. 


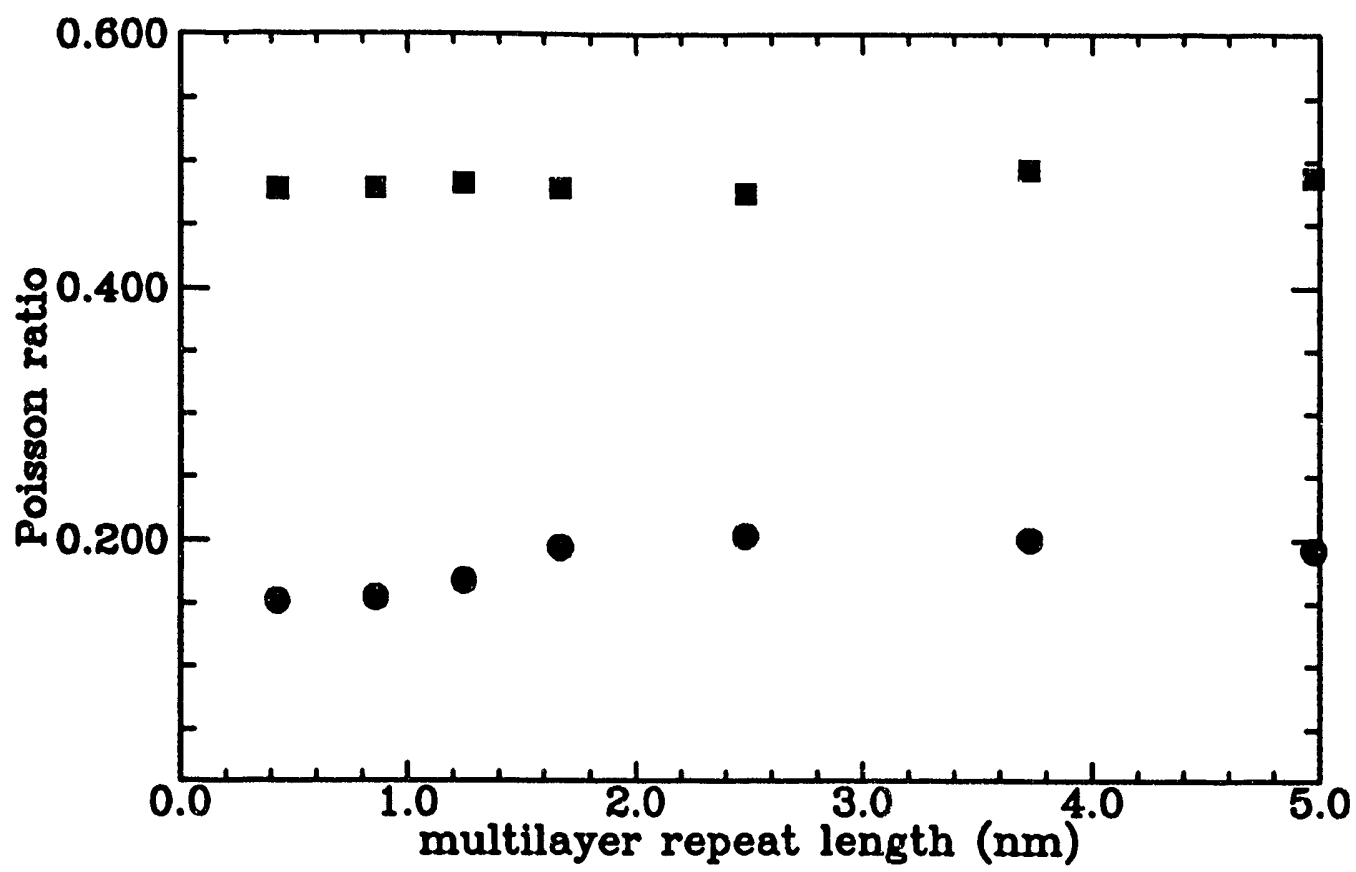

Figure 3. The calculated in-plane (squares) and out-of-plane (circles) Poisson ratios as a function of multilayer repeat length for uniaxial stress along the $x$ axis. The in-plane Poisson ratio appears to be insensitive to repeat length and the decrease in the out-of-plane ratio below repeat lengths of $1.5 \mathrm{~nm}$ appears to be significant.

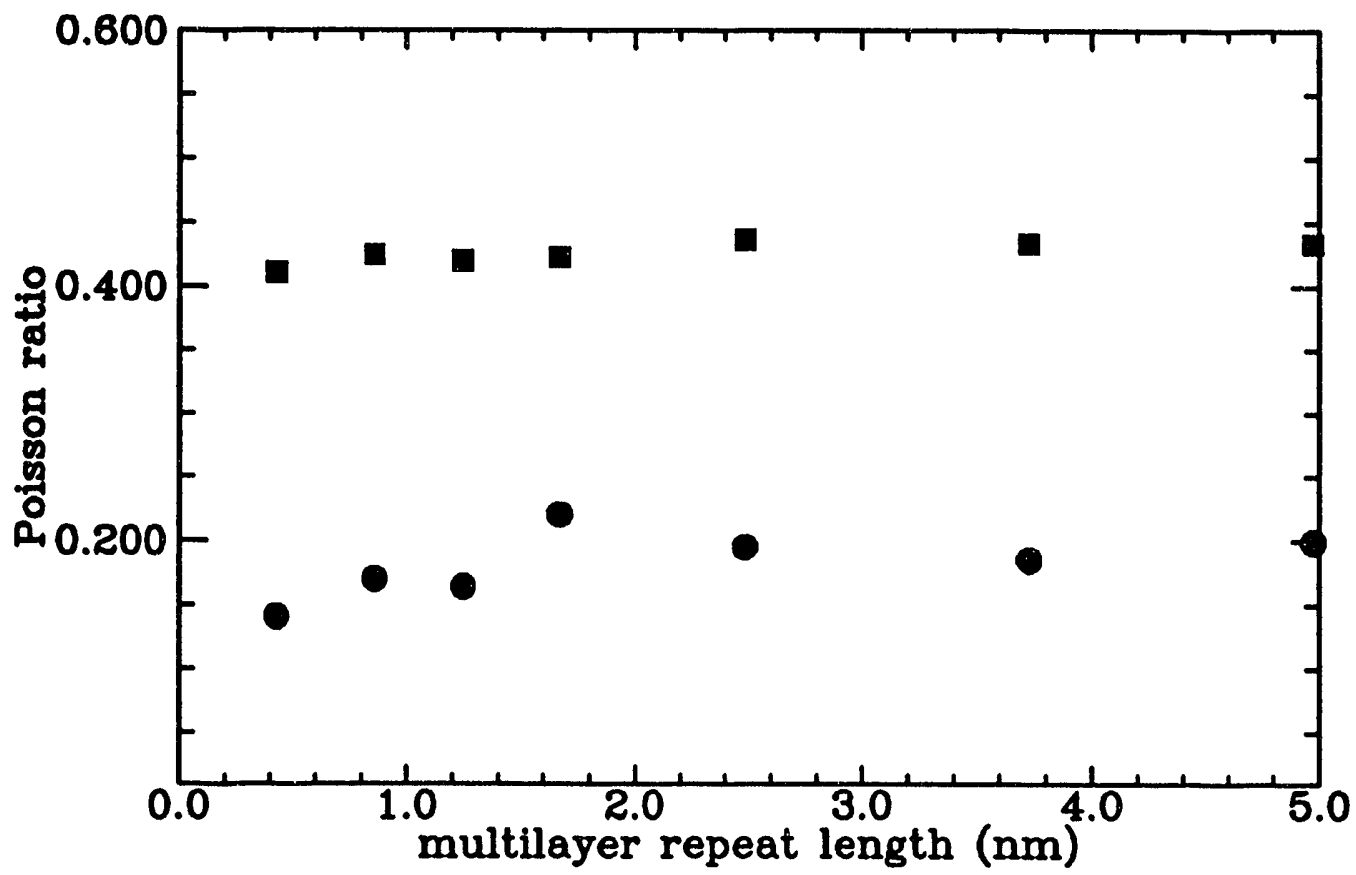

Figure 4. The calculated in-plane (squares) and out-of-plane (circles) Poisson ratios as a function of multilayer repeat length for uniaxial stress alo' ratio appears to be insensitive to repeat length and the decreas? in the out-of-plane ratio below repeat lengths of $1.5 \mathrm{~nm}$ appears to be significant. 


\section{RESULTS}

The calculated average interlayer spacing (d) is shown in Figure 1 as a function of multilayer repeat length $(n)$ for zero applied stress. The large oscillations in $d$ for $n<2 \mathrm{~nm}$ are beyond the statistical uncertainty in the simulations. These oscillations are due to the interlayer bonding. For $\mathrm{n} \sim 0.4 \mathrm{~nm}$ (a repeat length of 2 layers), each $\mathrm{Cu}(\mathrm{Ni})$ layer is sandwiched between $2 \mathrm{Ni}(\mathrm{Cu})$ layers. For $\mathrm{n} \sim 0.8 \mathrm{~nm}$, a $\mathrm{Cu}(\mathrm{Ni})$ layer feels a $\mathrm{Cu}(\mathrm{Ni})$ layer on one side and a $\mathrm{Ni}(\mathrm{Cu})$ layer on the other side, with a predicted additional repulsion between the layers. For $n \sim 1.2 \mathrm{~nm}$ some $\mathrm{Cu}$ (Ni) layers are completely surrounded by $\mathrm{Cu}(\mathrm{Ni})$ layers and the interlayer repulsion goes down. This effect continues until $n>2 \mathrm{~nm}$, afterwhich the further layers do not communicate due to the short range nature of the Johnson potential model (each layer interacts with the first two layers above and below).

The calculated elastic moduli (Y) for uniaxial tensile stress along the $\hat{x}$ (close-packed) axis and $\hat{y}$ axis in the simulation cell are shown in Figure 2 as a function of multilayer repeat length. To within the statistical uncertainty in the simulation, we observe no dependence of elastic moduli on multilayer repeat length, though we note a consistently higher modulus for applied stress along $\hat{y}$.

The calculated in-plane and out-of-plane Poisson ratios for applied tensile stress along the $\hat{x}$ axis is shown in Figure 3 and for applied stress along the $\hat{y}$ axis is shown in Figure 4. The slightly smaller in-plane ratio for applied stress along the $\hat{y}$ axis is consistent with the slightly larger elastic modulus. Most notable from these figures is the significantly smaller out-of-plane ratio than the in-plane ratio. Both of the out-of-plane ratios display a decrease in value, on the order of the uncertainty in the calculation, for $\mathrm{n}<1.5 \mathrm{~nm}$. This decrease correlates with the increase in average interlayer spacing for $\mathrm{n}<2 \mathrm{~nm}$ shown in Figure 1.

\section{DISCUSSION}

Comparison with previous simulation[9] is not possible due to the (001) stacking direction used in the previous work and comparison with experiment is difficult due to the ideal nature of the simulated multilayers. Defects in real multilayers come in several flavors:

(a) Stacking faults and other surface/interface defects.

(b) Dislocation loops.

(c) Interlayer diffusion, vacancies and other point defects.

Including these effects is complicated by two factors: (1) the embedded-atom method underestimates the stacking fault energy in fcc metals by an order of magnitude and (2) the specification of the system corresponding to experimental conditions may not correspond to equilibrium conditions.

There are other uncertainties associated with the simulation. For example, the applied stress $(\sim 1.6 \mathrm{GPa})$ and resulting strain $(\sim 1 \%)$ in these simulations is quite large. Even as such, the statistical uncertainty in the calculated elastic moduli is several percent for runs of length 10000 time steps (4-5 significant figures in the average cell lengths $\left(\mathrm{h}_{\mathrm{ij}}\right)$ and 2-3 significant figures in the differences $\left.\left(\varepsilon=\Delta h / h_{0}\right)\right)$.

Subject to these limitations, the simulations presented here display some interesting effects. The oscillations in the average interlayer spacing for small multilayer repeat lengths is probably very sensitive to the nature of the alloy potencial model. Clearly more precise quantum mechanical calculations and careful experimental measurements using x-rays or neutrons are required to determine whether the effects are real.

\section{ACKNOWLEDGEMENTS}

*Work performed at the Lawrence Livermore National Laboratory under the auspices of the U.S. Department of Energy pursuant to University of California-Lawrence Livermore National Laboratory Contract No. W-74()5-ENG-48. The authors gratefully acknowledge many enlightening discussions on multilayer materials with Troy Barbee Jr. and Tin Weihs and on atomistic materials modeling with Jim Glosli. 


\section{REFERENCES}

1. Multilayers: Synthesis, properties, and non-electronic applications, edited by T.W. Barbee Jr., F. Spaepen, and L. Greer (Mater. Res. Soc. Symp. Proc. 103, Pittsburgh, Pa 1988).

2. T. Tsakalakos and J.E. Hillard, J. Appl. Phys. 54, 734 (1983).

3. T.E. Schlesinger, R.C. Cammarata, C. Kim, S.B. Qadri and A.S. Edelstein in Thin Films: Stresses and Mechanical Properties II, edited by M.F. Doerner, W.C. Oliver, G.M. Pharr and F.R. Brotzen (Mater. Res. Soc. Symp. Proc. 188, Pittsburgh, Pa, 1990) p. 295.

4. M.S. Daw and Mi. Baskes, Phys. Rev B 29, 6443 (1984); S.M. Foiles, Phys. Rev. B 32, 3409 (1985).

5. D.J. Oh and R.A. Johnson in Atomistic Simulation of Materials: Beyond Pair Potentials, edited by V. Vitek and D.J. Srolovitz (Plenum Press, New York, 1989) p. 233.

6. R.A. Johnson, Phys. Rev. B 39, 12554 (1989).

7. M. Parrinello and A. Rahman, Phys. Rev. Lett. 45, 1196 (1980); J. Ray, J. Chem. Phys. 79, 5128 (1983).

8. C.L. Cleveland, J. Chem. Phys. 89, 4987 (1988).

9. J. Mei and G.W. Fernando, Phys. Rev. Lett. 66, 1882 (1991). 

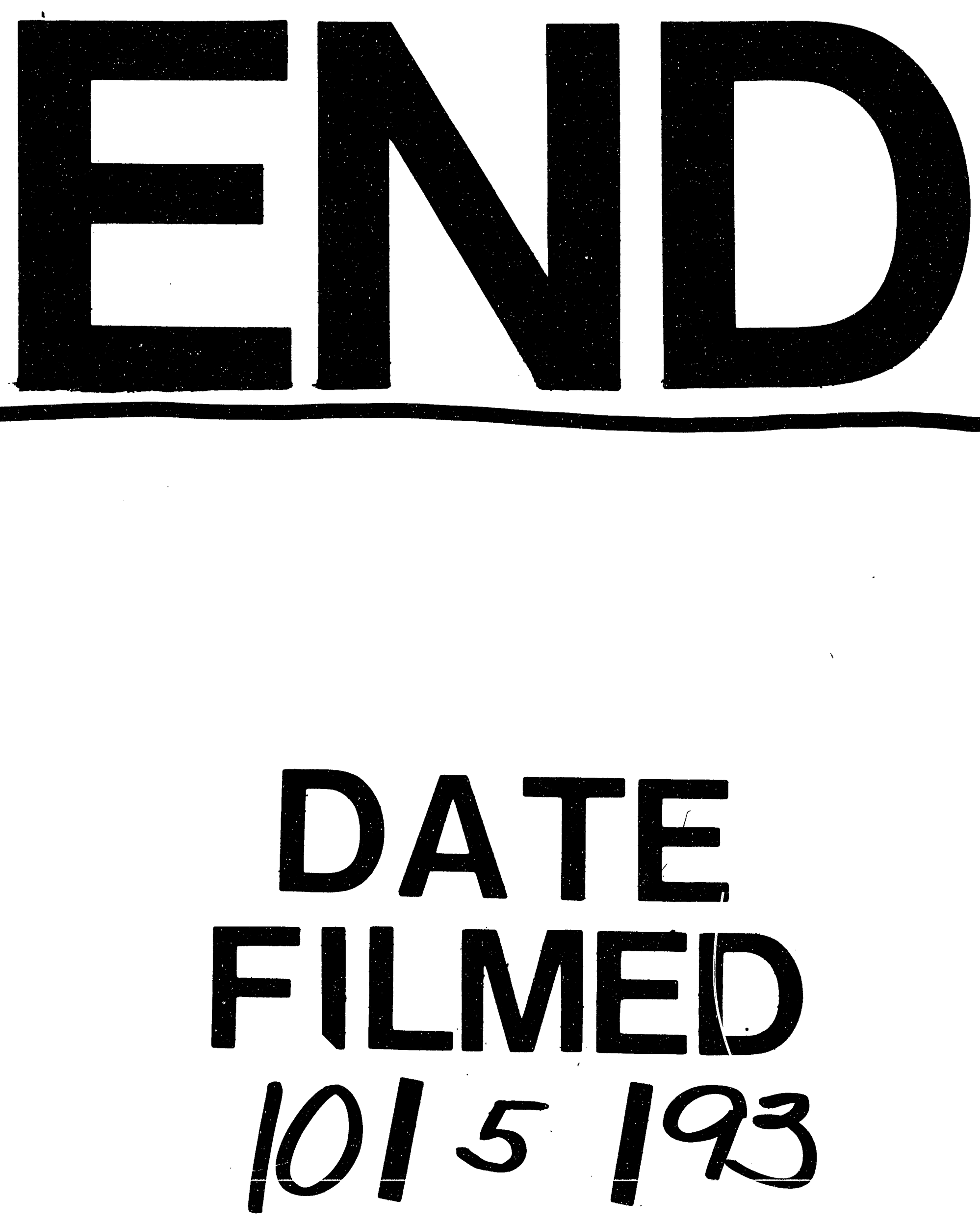
